\title{
Sitzung vom 24. Juli 1876.
}

Vorsitzender: Hr. A. W. Hofmann, Präsident.

Nach Genehmigung des Protocolls der letzten Sitzung werden die in derselben Vorgeschlagenen in Gemässheit des $\S 4 \mathrm{der}$ bisher gültigen Statuten zu Mitgliedern gewählt,

nämlich die Herren:

Baron N. v. Dellinghausen, Gut Kattintak, Station Katharinen der Baltiscben Eisenbahn.

Dr. F. Sehmuziger, Assistent an der medicinischen Klinik der Universität Zürich.

Carl Mann, Arnoldshöhe bei Cöln am Rhein.

W. F. A. Bohlen,

Jacob Zieg ler,

Th. Reinhart,

\section{Zürich}

Polytechuicum.

Die folgenden Herren, auf welcbe bereits die am 22. Juli 1876 in Kraft getretenen Statuten Anwendung finden, haben sich zur Aufnahme in die Gesellschaft gemeldet und sind als ausserordentliche Mitglieder vorgeschlagen, nämlich, die Herren:

Dr. Hermann Pfeiffer, Freiburgi.Br., v.d. HH. stud.phil.Richard Lindhorst, Claus u. Wichelhaus, Dr. Rudolf Köppen, Apotheker in Salzbrunn in Schlesien,

Dr. Adalbert Roch, Chemiker des landwirthsch. Instituts Halle a. $\mathrm{S}$.

Ruaolf Sachtleben, Halle a. S.

von den HH. Schmidt und Wichelbaus

Der Vorsitzende sagt, er freue sich, im Anschluss an die Mittheilungen, welche er hinsichtlich des Liebig-Denkmals in der letzten Sitzung gemacht habe, der Gesellschaft heute über die erwünschte Entwicklung dieser Angelegenbeit weiter berichten zu können.

Die Aufnabme, welche der Vorschlag des Vorstandes, die Liebig Statue sowohl in Giessen wie in München aufzustellen, gefunden habe, sei eine äber alle Erwartung günstige gewesen. Bisher hätten von den 139 Mitgliedern des General-Comités 96 eine directe Antwort an deu Vorstand gelangen lassen. Alle diese Antworten, ohne Ausnahme, sprächen rückhaltlose Zustimmung zu dem Vorschlag des Vorstandes aus, viele in Ausdrücken der lebhaftesten Befriedigung. Von ganz besonderer Bedeutung aber für die gedeibliche Entfaltung der Sache sei ein Schreiben aus München, welches noch kurz vor der Sitzung eingelaufen sei. Er habe bereits in der letzten Sitzung er- 
wähnt, dass das Münchener Comité dem Vorschlage des Vorstandes, so weit derselbe die Aufstellung derselb en Statue in München und Giessen betreffe, bisher seine Zustimmung versagt babe, dass aber die Fortsetzung der noch schwebenden Verbandlungen eine Verständigung auch über diesen Punkt erhoffen lasse. Diese Hoffnung sei bereits in Erfüllang gegangen, wie die Versammlung aus dem folgenden Schreiben ersehen werde.

Das Münchener Local-Comité für die Errichtung eines Liebig Denkmals in München

an den Vorstand der deutschen chemischen Gesellschaft zu Berlin.

Ihr Vorschlag, dass dem General-Comité das Recht der Reproduction des in München zu errichtenden Denkmals, behufs Aufstellung eines Denkmals in der Stadt Giessen gewahrt werde, wurde in unserer Sitzung am 13. d. M. einer erneuten Berathung unterzogen, und beehren wir uns Ihnen das Ergebniss dieser Berathung nachstehend mitzutheilen.

Das hiesige Comité hat beschlossen:

„Unter Bezugnahme auf seine schon früher ausgesprochene Zustimmung zur Verwendung von $25000 \mathrm{M}$. aus den für das Liebig-Denkmal gesammelten Beiträgen behufs Errichtıng eines Liebig-Denkmals in Giessen, erklärt das Münchener Comité sich auch mit dem weiteren Vorschlage, die Benutzung der Modelle des Münchener Denkmals für das in Giessen zu errichtende Denkmal betreffend, wie er nach Besehluss des Vorstandes der deutseben chemiseben Gesellschaft zu Berlin vom 25. Juni d. J. den Mitgliedern des General-Comités sub No. II. unterbreitet wurde, einverstanden."

Wir bemerken bierzu ausdrücklich, dass mit diesem Beschluss das hiesige Comité als Ganzes seine Zustimmung zu den jenseitigen Vorseblägen erklärt hat, dass dieser Beschluss somit die Zustimmung aller Mitglieder des hiesigen Comités, auch derjenigen, welche in der betr. Sitzung nicht anwesend waren, einschliesst.

München, 22. Juli 1876.

Im Auftrage des Münchener Comités

der Vorsitzende:

der Schriftführer:

Reichsrath von Niethammer,

J. Volhard.

Hiermit sei die vollständige Uebereinstimmung der Ansichten des Münchener und des Berliner Comités, auf welche der Vorstand so grosses Gewicht lege, zur Thatsache geworden.

Er wolle nur noch darauf hinweisen, dass, wie dies der letzte Paragraph des Münchener Schreibens auch besonders betone, der Beschluss des Münchener Comités für sämmtliche Mitglieder desselben bindend sei. Da nun dieses aus 23 Mitgliedern bestehe, unter den bereits erwähnten 96 zustimmenden Antworten aber bis jetzt keine aus München eingelaufen, so dürfe er das erfreuliche Ergebniss verzeichnen, dass von 139 Mitgliedern des General-Comités, $96+23=119$, dem Vorschlage der Gesellschaft bereits ihre Genebmigung ertheilt hätten. Wenn man bedenke, dass mehrere Mitglieder dieses Comités 
in Amerika wohnten oder sich im Augenblicke dort aufhielten, wenn man sich ferner erinnere, dass dies die Zeit der Reisen und Badekuren sei, so würde man zugeben müssen, dass der Vorstand alle Ursache habe, mit der Aufnahme, die sein Vorschlag gefunden, zufrieden zu sein. Der Verwirklichung desselben dürfe wohl jetzt keine erhebliche Schwierigkeit mehr im Wege stehen.

Die Gesellschaft werde schliesslich mit Genugthuung erfahren, dass der Vorschlag des Vorstandes zumal auch in der Stadt Giessen mit lebhafter Freude begrüsst worden sei. Das folgende Schreiben des Bürgermeisters Hrn. A. Bramm lege hierfür ein beredtes Zeugniss ab:

$$
\text { Giessen, den 14. Juli } 1876 .
$$

An

den Vorstand der deutschen chemischen Gesellschaft

in Berlin.

Nachdem der Stadtvorstand in Giessen Kenntniss davon erhalten, mit welchem Eifer und Erfolge der hochgeehrte Vorstand der deutschen chemischen Gesellschaft zu Berlin dafür gewirkt bat, dass ein Theil der für die Errichtung eines Liebig-Denkmals gesammelten Beiträge zur Aufstellung eines solchen auch in hiesiger Stadt verwendet werde, hat derselbe in seiner gestrigen Sitzung beschlossen, diesem bochverehrlichen Vorstande seinen innigsten Dank für dessen Bemühungen auszudrücken, und rechnet es sich der ganz ergebenst Unterzeichnete zur besonderen Ehre, dass ihm der Auftrag ertheilt worden ist, diesen Dank hiermit auszusprechen.

Mit ausgezeichneter Hochachtung

zeichnet

A. B ram m,

Bürgermeister.

Die nächste gemeinsame Aufgabe, welche dem Münchener Comité und dem Vorstande der chemischen Gesellschaft obläge, müsse die Condensation des vielköpfigen General-Comités in ein werkfähiges Executiv-Comité sein. Er werde nicht ermangeln die Gesellschaft über die weitere Entwickelung der Sache au courant zu halten.

Der Vorsitzende erwähnt ferner, dass nach einer ihm von Hrn. Biedermann gemachten Mitheilung Hr. Dr. Hugo Müller in London die folgenden älteren Werke:

1) Erdmann's Journal für praktische Chemie. Jahrg. 1853-64.

2) Chemisches Centralblatt. Jahrg. 1858_-64

3) Dumas Handbuch der Chemie, dentsche Ausgabe. Bd. 5, 6, 7, 8.

4) Berzelius Chemie, ubersetzt von Wöhler, 1. Auflage.

5) Dana, Mineralogy.

6) Reichenbach, das Kreosot.

der Bibliothek der Gesellschaft zum Geschenk gemacht habe. Er spricht Hrn. Hu go Müller den Dank der Gesellschaft aus. Er hebt bei dieser Gelegenheit hervor, wie sehr die Bibliothek der Gesellschaft 
sich dazu eignet, eine Sammelstelle für seltene chemisch wissenschaftliche Werke und solche von historischem Interesse zu werden und fordert die Mitglieder auf, nach Kräften zu der Bereicherung der Bibliothek in diesem Sinne beizutragen.

Hr. Scheibler begrüsst das von Hrn. Dr. Mü1legr gegebene Beispiel als ein sehr nachahmungswerthes und nimmt Veranlassung, ein in seinem Besitz befindliches werthvolles Werk, Stahl's Nova elementa chemiae, der Bibliothek zu überweisen.

Für die Bibliothek sind ausserdem eingegangen bis zum 24. Juli: Als Geschenk:

Sur le developpement de l'électricité statique; par M. Walthère Spring. Bruxelles 1876 .

Ueber die Einwirkung einiger Metallbasen auf Monochloressigsïure. Inauguraldiss. von Gerhardt Schreiber. Leipzig 1876.

Im Austausch:

American Chemist Vol. VI, No. 10 und 11.

Bulletin de l'Académie Royale de Belgique. T. 41, No. 5 .

Bulletin de la Société chimique de Paris. T. XXVI, No. 2.

Bulletin de la Saciété industrielle de Rouen No. 3. Mai.

Chemisches Centralblatt. No. 28, 29,

Deutsche Industriezeitung. No. 28 u. 29.

Journal der Russischen chemischen Gesellschaft. T. VIII, No. 6.

Maandblad voor Natuurwetenschappen. No. 9.

Proceedings of the philosophical Society of Glasgow, Vol. $X$, No. 1.

Revue scientifique. No. 3 u. 4.

Durch Kauf:

Comptes rendus. T. LXXXIII, No. 1 a. 2.

Dingler's polytechnisches Journal. Bd. 221, Heft 1.

\section{Mittheilungen.}

\section{L. F. Nils on: Zur Erage über die Valenz der seltenen Erdmetalle.}

(Dritte Mittheilung.)

(Eingegangen am 12. Juli; verlesen in der Sitzung von Hrn. Se11.)

Zar Ergänzung meiner letzten Mittheilung will ich, betreffend die Chloroplatinate der vierwerthigen Elemente, zuerst erwätituen, d’ass es mir nachher gelungen ist, zwei Verbindungen ron Zinn und Zirkonium darzustellen. Sie krystallisirten aus einer Mischung von $1 \mathrm{Mol}$. der freien Chlorosäure und 1 Mol. Zinntetrachlorid oder Zirkoniumoxychlorid, nachdem die Chlorwasserstoffsäure durch Abdampfen im Wasserbade ausgetrieben war. Die neuen Verbindungen sind:

Stannichloroplatinat: $\quad \mathrm{Sn} \mathrm{Cl}{ }^{4} \cdot \mathrm{Pt} \mathrm{Cl}^{4}+12 \mathrm{H}^{2} \mathrm{O}$, in klejnen, glätuzenden, hellgelben, dünnen, vierseitigen, vielleicht etwas schiefen Tafeln krystallisirend [gef. $13.94-14.98 \mathrm{Sn}, 24.75-$ 24.79 Pt. statt resp. 14.46 und 24.27]. 